\title{
Design and Emotional Expressiveness of Gertie (An Open Hardware Robotic Desk Lamp)*
}

\author{
Fabian Gerlinghaus ${ }^{1}$, Brennand Pierce ${ }^{2}$, Torsten Metzler $^{3}$, Iestyn Jowers ${ }^{4}$, Kristina Shea $^{5}$ and Gordon Cheng ${ }^{6}$
}

\begin{abstract}
This paper introduces Gertie the Robotic Desk Lamp, a novel research platform that has five degrees of freedom, and is equipped with a camera and microphone in its lamp shade. These features mean that Gertie is a flexible and low-cost resource for conducting research into cognitive products and human-robot interaction. It will be available as an open hardware on http://www.opengertie.org/.

Gertie was designed from first principles, and assembled using off the shelf electronic components and parts fabricated using a 3D printer. In this paper, the design of Gertie is presented, and its application as a research platform is described. Gertie has already been used to investigate a problem of simple object tracking, building on computer vision algorithms. Furthermore, it has also been used to investigate and replicate emotional body language.

By imitating human body language Gertie is capable of expressing four of the basic Ekman emotions: 1) joy; 2) sadness; 3) surprise; and 4) fear. This work was validated using an online study, which investigates how well the emotions expressed by Gertie are recognized by human audiences. In total 84 participants were shown one video for each of the four emotions and they were asked to choose from a list of seven emotions, which they thought was displayed by Gertie. While joy and sadness were recognized very reliably with $81 \%$ and $88 \%$ of all people giving the correct answer, fear and surprise were more commonly misinterpreted as surprise and disgust. However, all emotions were recognized above the chance level percentage of $14 \%$.
\end{abstract}

\section{INTRODUCTION}

Personal robots will play an ever more important role in our society, for example, in assisting the elderly. Since a large part of human communication takes place non-verbally, there has been considerable research investigating robots capable of communicating emotions. Several examples include the robotic heads Kismet [1], Eddie [2] and Mask-Bot [3], which display emotions by means of facial expressions. Kismet is a very sophisticated social robot developed at the MIT in the 1990s. Eddie is a simpler alternative with a strong focus on displaying emotions, developed at the Technical University of Munich (TUM). Kismet and Eddie use mechanical parts for animating elements of the face like lips, eyes or eyelids. In contrast, Mask-Bot employs a semitransparent face mask

* This work was supported by the Institute for Cognitive Systems, http://www.ics.ei.tum.de/, Technical University Munich, Karlstrasse 45, 80333 Munich, Germany; as well as by the Virtual Product Development Group, http://www.pe.mw.tum.de/, Technical University Munich, Boltzmannstr. 15, 85748 Garching, Germany.

1 fabian.gerlinghaus@cdtm.de

2 brenetum.de

3 torsten.metzlerepe.mw.tum.de

4 iestyn.jowersepe.mw.tum.de

5 kristina.shea@pe.mw.tum.de

6 gordondtum.de

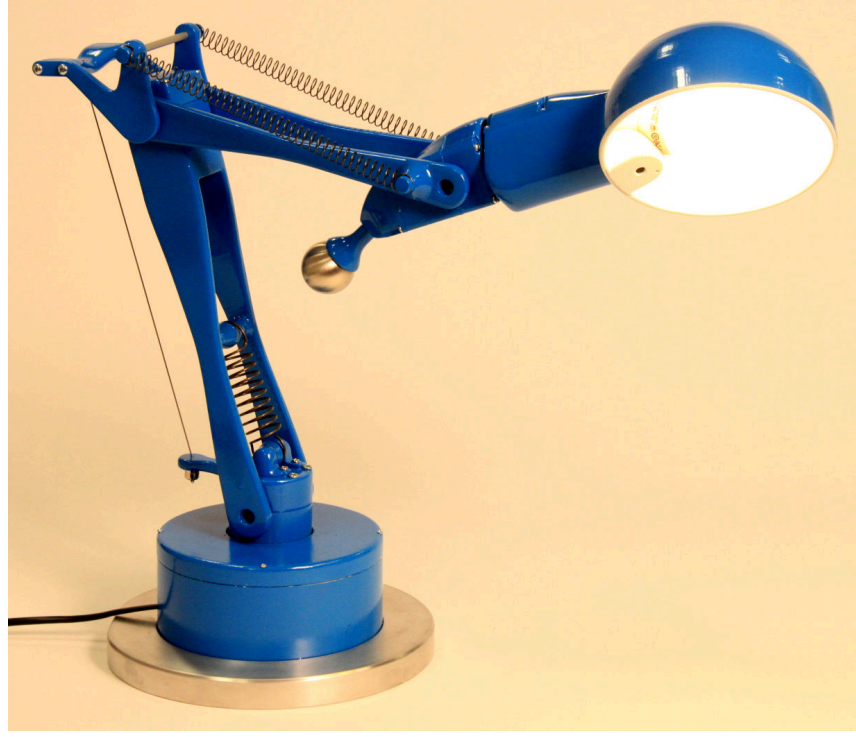

Fig. 1. Gertie, the Robotic Desk Lamp.

as a screen for a talking head animation engine that is broadcast from the projector mounted behind the mask. This way the head becomes a life-size talking head in real space as opposed to 2D flat screen space [3], [4].

Other research focused on whole-body humanoid robots, like the NAO humanoid robot [5], which communicates emotions by means of body language. Another whole-body humanoid robot named Kobian was developed by Zecca et al. [6]. In contrast to the other robots Kobian is capable of displaying emotions both by body language as well as by facial expressions. The research on Kobian shows, that the body alone or the face alone do not have the same expressiveness that the body plus the face can reach, thus showing the importance of a holistic approach.

Closely related to our work, are the works of Hoffman [7] and Kozima et al. [8]. Hoffman created AUR, a robotics desk lamp that target the studies of quality of Human Robot Interaction (HRI), whereas the works of Kozima et al., target the clinical child psychology studies.

This paper presents Gertie the Robotic Desk Lamp. While most of the robots mentioned above imitate human appearance, Gertie looks like a common desk lamp and unlike most robots which have many degrees of freedom (DOF), Gertie only has five DOF. Despite its simplicity, it is capable of expressing emotions, as will be shown by a study presented in this paper. 
Gertie was inspired by the Pixar short film "Luxo Jr."1 which stars two desk lamps that move and behave as if they had a personality. The long term vision for this robot is to create a convincing illusion of a cognitive lamp that interacts with humans via speech recognition and by displaying emotions.

Additionally Gertie can serve as a versatile research platform for Cognitive Products [9]. It will be available as an open hardware on http://www.opengertie.org/. This provides students and research institutions with a cost-effective alternative for investigating human-robot interaction and many other fields of research.

Section II of this paper, describes the design and fabrication of Gertie. Section IV suggests several applications of Gertie as a research platform. Gertie has been used to investigate and replicate emotional body language. Furthermore, a survey on the recognizability of these emotions was conducted. This research is presented in Section III.

\section{DESIGN AND IMPLEMENTATION}

\section{A. DESIGN}

All mechanical parts were modeled with SolidWorks by Dassault Systèmes ${ }^{2}$. They were 3D printed, out of ABS plastic using the fused deposition modeling machine Stratasys $1650^{3}$. An important design criterion was to hide all electronic components, in order to create the impression of a common desk lamp. In its default configuration the lamp has approximately the following dimensions: $550 \mathrm{x} 420 \mathrm{x}$ $220 \mathrm{~mm}$ (see Fig. 1).

Gertie's actuators and sensors are connected to the USB port of a PC, which performs all the computation. This PC runs Ubuntu Linux Operating System. C++ and the Robot Operating System $\left(\mathrm{ROS}^{4}\right)$ are used for controlling the servo motors and the OpenCV library is used for vision processing and object tracking.

\section{B. GRAVITY COMPENSATION}

The Robotic Desk Lamp was designed to be manually moved into an arbitrary posture when the motors are off. It keeps this posture despite the force of gravity due to a counterweight, as well as two spring mechanisms which are used for gravity compensation, as shown in Fig. 2 . Gravity compensation was important for achieving the same dynamic movements with small motors, while providing the functionality of a common desk lamp.

Only joints 2, 3 and 4 are affected by gravity. Fig. 2 shows that joint 2 and 3 are balanced by springs. To determine the gravitational effects acting on the lamp, a MATLAB model was created of the mechanical structure, distances and spring properties could be dimensioned, such that the torque created by a spring equals the torque created by the force of gravity for every angle of the respective joint.

\footnotetext{
1 "Luxo Jr." by Pixar Animation Studios (1986)

${ }^{2}$ http://www.3ds.com/

${ }^{3}$ http://www.stratasys.com/

${ }^{4}$ http://www.ros.org/
}

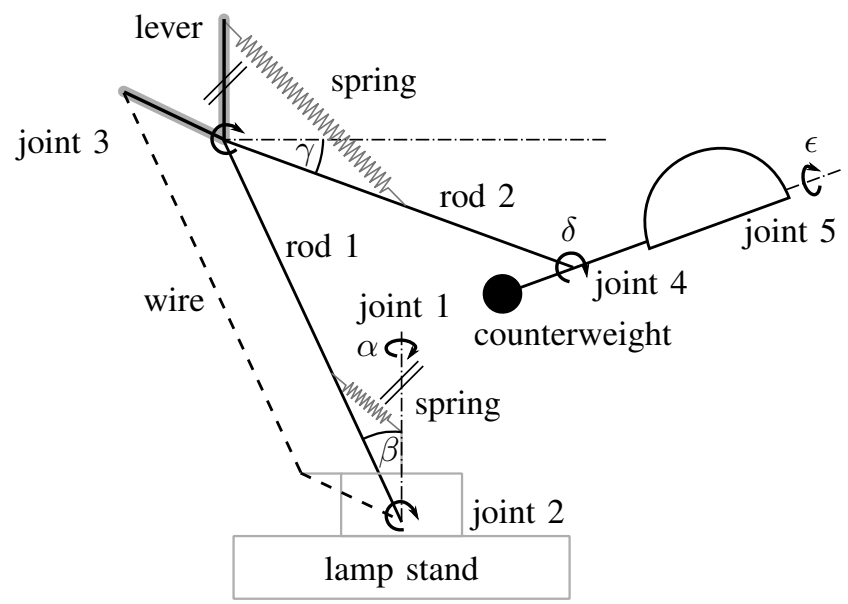

Fig. 2. Gravity Compensation Mechanisms.

The gravity compensation mechanisms for joints 2 and 3 need to be independent of each other. Therefore the spring balancing joint 3 is attached to the vertical arm of the lever. Due to the parallelogram structure of lever, wire and rod 1 the right arm of the lever always keeps its vertical orientation independent of the angle $\beta$.

Joint 4 is balanced by a counterweight which also serves as a knob for manually positioning the lamp, when the motors are off.

\section{PART SELECTION}

Once the kinematic structure of the lamp was defined and the dynamics of the fastest lamp movement were approximated, the required motor torque and the required angular velocity were calculated to be: $M_{\min }=0.70 \mathrm{Nm}$ and $\omega_{\min }=\pi \frac{\mathrm{rad}}{\mathrm{s}}$. These calculations neglected friction as well as gear and motor inertia and assumed that the gravity compensation mechanisms were working perfectly. Based on these requirements the Dynamixel AX-12A servo was chosen which provides twice the necessary torque. Another advantage of these motors is their digital interface, which provides feedback, for example position and temperature and current. They can easily be connected to the USB port of a PC and drivers were already available within ROS. Gertie is equipped with a small USB-Webcam that is integrated below the light bulb in the lamp shade, as can be seen in Fig. 1. The Webcam can provide color images with a resolution of $360 \times 480 \mathrm{px}$ at 30 FPS. A microphone is integrated in the camera. Just like the servo controller the camera is connected to a USB hub in the lamp stand.

\section{STRUCTURAL ANALYSIS}

The mechanical structure of the lamp is required to be very stiff in order to avoid undesirable oscillations. For simplicity the rods were directly attached to the servo motors. Additional bearings would have made joints even stiffer, but the design would have become undesirably large.

Angular tolerance in the axis of the joints is a bigger problem the earlier it appears in the kinematic chain. If for example the $\alpha$-axis of joint 1 has a slackness of $\pm 1^{\circ}$ this 


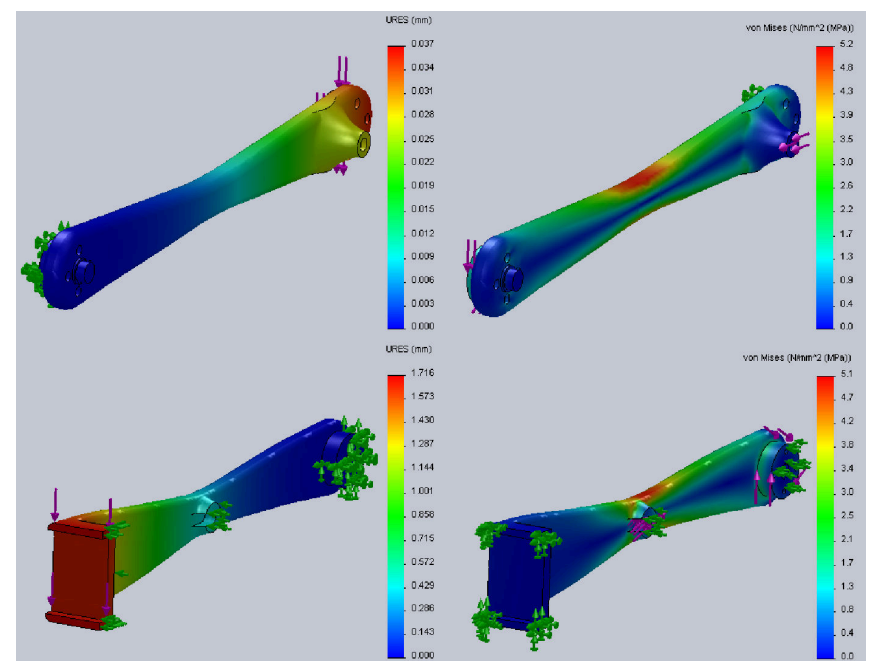

Fig. 3. FEM Simulation of the Connecting Rods Under Load.

creates a slack of $\pm 8 \mathrm{~mm}$ in the position of the end effector. The same deviation in the $\epsilon$-axis of joint 5 creates a slack of less than one millimetre in the position of the end effector. For this reason the axis of the first joint was equipped with an additional bearing, which is integrated in the cap of the lamp stand. This significantly increased the rigidity of the structure.

The most stressed parts in the whole mechanical structure are the connecting rods between joints 2 and $3(\operatorname{rod} 1)$ and in between joints 3 and 4 (rod 2), see Fig. 2. For this reason special attention was given to their dimensioning. Due to the high cost of rapid prototyping material as well as for aesthetic reasons, the rods are designed to use as little material as possible while while at the same time maintaining the necessary strength.

Each rod is required to withstand the maximum mechanical strain created by the spring and the servo motor. Additionally, the elastic deformation under load may not exceed one percent of the rods length. To meet these requirements an finite element method (FEM) simulation was carried out for each of the rods. Fig. 3 shows rod 1 on the bottom and rod 2 on top. On the left side of Fig. 3 the displacement under maximum mechanical strain is shown for each of the rods. On the right side the von Mises stress is depicted. The von Mises theory calculates a scalar stress value from the three dimensional stress tensor, which can be compared to the tensile strength of the material. The FEM simulation showed that the von Mises stress of both parts reaches a maximum value of $5 \frac{\mathrm{N}}{\mathrm{mm}^{2}}$ in the middle of the rods. The tensile strength of Fused Deposition Modeling ABS is $35 \frac{\mathrm{N}}{\mathrm{mm}^{2}}$. Consequently, both parts will bear the stress with a security factor of $S=\frac{35}{5}=7$. This value proved to make the structure sufficiently rigid to avoid oscillations.

\section{INVESTIGATING HUMAN-ROBOT INTERACTION WITH GERTIE}

\section{A. IDENTIFYING AND REPLICATING EMOTIONAL BODY LANGUAGE}

In the context of the ageing population of Europe and Japan, robots are expected to play an important role in our society assisting elderly people [10]. Additionally, many robots are developed for entertainment. A good example for this is the dancing Sony QRIO robot. For both of these application domains, Human-Robot Interaction (HRI) is essential. Since a large part of human communication takes place non-verbally, extensive research is being conducted on providing robots with facial expressions and body language to increase their acceptance among humans and make HRI more intuitive and entertaining.

1) Anthropomorphisms: Much of the research mentioned above is conducted on humanoid robots like Kobian [6] and NAO [5]; as well as well as on humanoid heads like Kismet[1] or Eddie [2]. Because of the close resemblance to humans these robots make it relatively easy for a human observer to decode the emotions displayed by these robots.

However, the look of a robot can strongly differ from human appearance and its body language can still be understood easily. This is because of the human peculiarity to anthropomorphize. The first study of this phenomenon was by Charles Darwin [11]. Later the studies presented by Caporael confirmed that humans constantly anthropomorphize animals and inanimate objects [12].

According to Blake and Shiffrar[13] the human visual system is tuned for the pickup of socially relevant information and it needs surprisingly little information to do so. They investigated point light videos, in which small light tokens are attached to the limbs of a human actor. The videos only show these light points, not the actor himself. Experiments showed that a point light video of an arm knocking against a door was enough information to determine the emotional state of the actor.

In 1986 the Pixar Animation Studios created a short film called "Luxo Jr." The only characters are two Anglepoise desk lamps, one large and one small. Luxo Jr. (small) is playing with an inflated rubber ball, chasing it and trying to balance on it, as Luxo (large) reacts to these antics. The ball eventually deflates due to Luxo Jr. jumping on it. Luxo Jr. is admonished by Luxo, who then finds Luxo Jr. playing with an even larger ball.

Anthropomorphisms are the underlying principle of this short film. The shapes of the lamps in the movie have very little resemblance to a human, but the audience nonetheless interprets its lampshade as a head and the light bulb as an eye. The movie only shows two lamps which move, but humans interpret these movements as a body language revealing information about the emotional state of the lamps.

If we are to anthropomorphize Gertie in much the same way as the lamps in "Luxo Jr.", its lamp shade is the equivalent of a head, the light bulb resembles an eye and the lever above joint 3 represents the shoulders. Additionally, 
rod 2 corresponds to its upper body, rod 1 is interpreted as Gertie's legs and the base plate is interpreted as Gertie's feet. These anthropomorphisms are the basis for the development of Gertie's emotional body language.

Next to this the importance of giving a name to the lamp may not be underestimated, because it encourages humans to anthropomorphize. The Robotic Desk Lamp was named Gertie, in honour of one of the the first animated short films "Gertie the Dinosour" from 1914.

2) Emotion Models: In psychology there are two popular models which systemize human emotions. Russel describes emotions as a point in space spanned by three axes: pleasure, arousal and dominance (PAD) [14]. Paul Ekman categorized all human emotions in six basic emotions: 1) anger, 2) disgust, 3) fear, 4) happiness, 5) sadness and 6) surprise [15].

Russells PAD model was used to create an affect space for the robotic head Eddie [2], as well as for a NAO robot [5]. The advantage of this model of emotions is that it allows smooth transitions from one emotion to another by planning a trajectory through affect space over time. However, each emotion is represented by a static pose of the NAO humanoid robot or by a certain configuration of all of the variables that define the facial expression of Eddie.

Gertie only has five degrees of freedom - considerably less than, Eddie or a NAO. It also does not resemble humanoids. These are two factors which constrain its expressiveness. However, it has been shown that motion provides even more reliable and compelling information about the affective state than static pictures [13]. This is why in contrast to the works on Eddie and NAO, dynamic movements were developed for the desk lamp to communicate emotions instead of static poses. These movements express four of the basic Ekman emotions: fear, happiness, sadness and surprise.

3) Human Body Language: The research on human body language was reviewed with a particular focus on the four emotions, that are to be expressed by Gertie. Charles Darwin investigated how emotions are expressed in the body language of men and animals [11]. He found that happiness is often expressed, by laughter, various purposeless movements and jumping, while the body is held erect and the head is in an upright position. In contrast sadness is expressed by slow, passive motion and the head hanging on the contracted chest.

Later a study by Beck et al. [5] confirmed that the head position is an important body posture variable. Happiness is connected to an upright head position, while a hanging head is associated with sadness or anger. This is especially important for the development of motion patterns of the Robotic Desk Lamp, since its lamp shade is anthropomorphized as a head. The rods that are connecting the base with the lamp shade are perceived as the lamp's body. A contracted chest can thus be represented by a pointed angle between the rods while an upright body position can be achieved by an obtuse angle. Fig. 4 illustrates these postures.

Darwin also states that the body language of someone experiencing fear is most often characterized by the attempt to retreat [11]. The whole body is rapidly turned away and

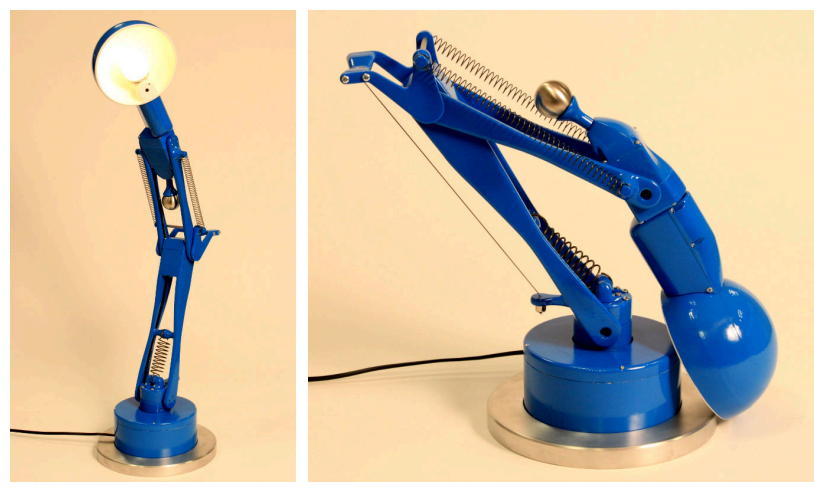

Fig. 4. Upright Position Contrasted with Contracted Position.

TABLE I

DESCRIPTION OF THE MOTION PATTERNS FOR EACH EMOTION.

\begin{tabular}{|l|l|}
\hline Happiness & $\begin{array}{l}\text { The head is bouncing up and down in fast movements. } \\
\text { The head is erect and looking in different directions } \\
\text { at the summit of each up phase. }\end{array}$ \\
\hline Sadness & $\begin{array}{l}\text { The lamp is moving very slowly as if it was very } \\
\text { heavy. The head is bent. Then the lamp is sighing } \\
\text { and lets its head sink even more, while its body is } \\
\text { folding into a contracted position. }\end{array}$ \\
\hline Fear & $\begin{array}{l}\text { The lamp is reacting to something very scary. While } \\
\text { always looking in the direction of this something, } \\
\text { the lamp is rapidly retreating. The angle between the } \\
\text { lamp shade and the second rod is chosen such that the } \\
\text { impression of the head sinking between the shoulders } \\
\text { is created. }\end{array}$ \\
\hline Surprise & $\begin{array}{l}\text { The lamp is scanning the environment with its gaze. } \\
\text { All of a sudden something that was just seen, but not } \\
\text { immediately grasped surprises it. The lamp rapidly } \\
\text { turns back its head in this direction. }\end{array}$ \\
\hline
\end{tabular}

the head sinks between the shoulders.

Moreover, this research was strongly influenced by the tremendous experience of animators in creating believable behaviour. In the 1930's animation grew from a novelty to an art form at the Walt Disney Studio. Ever since the same basic principles have been applied to classical as well as computer animation [16]. While some of these principles are very specific to animation, others proved to be very helpful for the development of motion patterns for the Robotic Desk Lamp. One of them is Timing. This principle states, that the speed at which an action is performed carries emotional meaning. Fast movements are associated with joy, aggression and surprise, while slow movements are associated with sadness.

Another principle that was taken into consideration is called Follow Through. It states that natural actions seldom come to a sudden end, but instead are carried out past their termination points. An example would be a hand throwing a ball, where the hand continues to move after releasing the ball.

4) Design of Motion Patterns: The research on human body language presented so far was used to develop four movements for the Robotic Desk Lamp. These movements imitate the way humans tend to move, when they feel happiness, sadness, fear or surprise.

Table I shows a description of the motion patterns. The most difficult emotion to display was surprise. Surprise is 
TABLE II

ForCED-CHOICE PERCENTAGE $($ RANDOM $=14.3 \%)$.

\begin{tabular}{|l|lllllll|}
\hline & Anger & Disgust & Fear & Joy & Interest & Sadness & Surprise \\
\hline Fear & $4.8 \%$ & $15.5 \%$ & $\mathbf{3 3 . 3 \%}$ & $0.0 \%$ & $1.2 \%$ & $0.0 \%$ & $45.2 \%$ \\
\hline Joy & $0.0 \%$ & $0.0 \%$ & $1.2 \%$ & $\mathbf{8 1 . 0} \%$ & $10.7 \%$ & $0.0 \%$ & $7.1 \%$ \\
\hline Sadness & $1.2 \%$ & $0.0 \%$ & $9.5 \%$ & $0.0 \%$ & $1.2 \%$ & $\mathbf{8 8 . 1 \%}$ & $0.0 \%$ \\
\hline Surprise & $17.9 \%$ & $28.6 \%$ & $16.7 \%$ & $1.2 \%$ & $10.7 \%$ & $0.0 \%$ & $\mathbf{2 5 . 0 \%}$ \\
\hline
\end{tabular}

mainly displayed in facial expressions, as discovered by Breazeal in her work on Kismet [1]. Since the Robotic Desk Lamp does not have facial expressions, another way of communicating surprise had to be found. In theatre the same problem exists. Often the audience is so far away from the actors, that facial expressions can hardly be recognized. Surprise is then often expressed by a bigger gesture - the double take - that can be seen from further away. A double take is defined as a rapid and surprised second look at a person or a situation whose significance had not been completely grasped at first.

5) Trajectory Planing: Four movements were developed, each of which communicates an emotion. This section describes how they were implemented.

In computer animation the standard procedure for planing and calculating trajectories of any moving object is to define key frames [17]. Each key frame defines a certain posture of the object at a certain time. The motion is generated by using splines to interpolate in between these key frames. While key frame animation could have been adapted for the Robotic Desk Lamp, it is a very abstract way of defining movements without proper software for the simulation and visualisation of the lamp's movements.

Instead, another approach was chosen which is called Programming by Demonstration [18]. In this technique Gertie is manually guided along the desired trajectory. Meanwhile all joint angles are recorded at $50 \mathrm{~Hz}$ and saved to a file. Afterwards the recorded trajectory can be replayed and the lamp moves precisely the way it was taught. This way the design of a new movement becomes very intuitive and fast.

\section{B. EVALUATING THE EMOTIONAL EXPRESSIVENESS}

After the movements for the four different emotions were implemented an online survey was conducted in order to evaluate how well the emotions are recognized by people who have not seen Gertie before. The survey was designed in the same way as Cynthia Breazeal's video survey on the recognizability of the emotions of Kismet to make the results comparable [1].

In total, 84 people participated in the survey on Gertie. All of them were shown four videos, each displaying one of the four different motion patterns. Afterwards they were asked to choose from a list of seven emotions, which they thought was displayed by the Robotic Desk Lamp in the video. The probability of choosing the right answer by chance is $\frac{1}{7}$. $100 \%=14.3 \%$. Participants were also asked how sure they felt about their decision on a likert scale from 1 to 5 , with 1 meaning very unsure and 5 meaning very sure.
TABLE III

RESULTS OF THE SURVEY.

\begin{tabular}{|l|llll|}
\hline & Fear & Joy & Sadness & Surprise \\
\hline Percentage correct & $33.3 \%$ & $81.0 \%$ & $88.1 \%$ & $25.0 \%$ \\
\hline $\begin{array}{l}\text { Most common } \\
\text { misinterpretation }\end{array}$ & $\begin{array}{l}\text { surprise } \\
(45.2 \%)\end{array}$ & $\begin{array}{l}\text { surprise } \\
(7.1 \%)\end{array}$ & $\begin{array}{l}\text { fear } \\
(9.5 \%)\end{array}$ & $\begin{array}{l}\text { disgust } \\
(28.6 \%)\end{array}$ \\
\hline $\begin{array}{l}\text { Average level } \\
\text { of certainty }\end{array}$ & 3.6 & 3.9 & 4.1 & 3.1 \\
\hline $\begin{array}{l}\text { Percentage correct in } \\
\text { survey on Kismet [1] }\end{array}$ & $86 \%$ & $57 \%$ & $86 \%$ & $71 \%$ \\
\hline
\end{tabular}

Table II shows which percentage of participants selected which emotion, after having seen the lamp move in the respective video. The correct answers are highlighted. As can be seen from the table each emotion was recognized by a percentage above the chance level of $14.3 \%$.

Table III summarizes the results of the survey. While joy and sadness were recognized very reliably with $81 \%$ and $88 \%$ of all people giving the correct answer, fear and surprise were more commonly misinterpreted as surprise and disgust. The average level of certainty also shows that people felt considerably less secure about their answers, when they interpreted the lamp's movements for fear and surprise. This might be due to the fact, that no context was given in the videos that were shown to the participants of the survey. While joy and sadness are emotions which do not necessarily have their cause in the physical world, fear and surprise are most often triggered as a reaction to events in the outside world. Consequently, the absence of context could have a stronger effect on the recognizability of the emotions fear and surprise.

The results of the survey on the recognizability of Gertie's emotions were compared with the results of Breazeal's survey on Kismet. Gertie communicates joy more reliably than Kismet. It is as good as Kismet when communicating sadness and worse when communicating fear or surprise.

In summary, the study shows that a "simple" Robotic Desk Lamp is capable of communicating emotions, despite the fact that it does not exhibit any explicit human characteristics and the fact that it only has five degrees of freedom.

\section{RESEARCH APPLICATIONS}

This section provides various examples of how Gertie can be employed in research as well as in education.

Due to the utilization of Programming by Demonstration teaching Gertie a new movement takes only a few seconds - even for people without any background in robotics. Psychologists for example could use Gertie for studies on body language or the nature of anthropomorphisms.

Furthermore, Gertie can be used to investigate how robots need to behave to create a compelling illusion of being alive and having a personality. Real living beings are never completely stiff - they breathe for example - and they never perform the same movement twice in the same numerically precise manner. Additionally, they recognize speech, gestures and faces. There are many challenges concerning cognitive products which can be investigated using the Robotic Desk Lamp. 


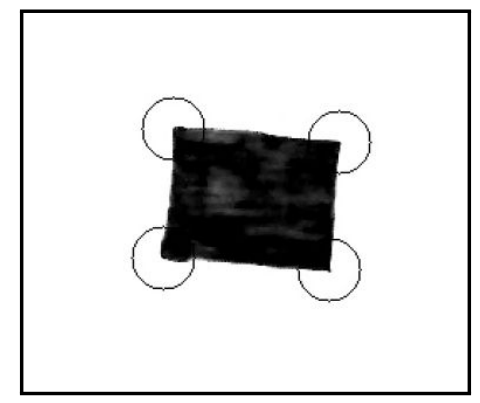

Fig. 5. Harris Edge Detection Algorithm.

Gertie is supposed to serve as an educational tool in handson university courses. Students could assemble the lamp and apply what they learned about inverse kinematics to a real robot. They could make it respond to vocal commands by including speech recognition software, or they could learn about computer vision by implementing a face tracking application for example.

In order to demonstrate how Gertie can be used as an education and research platform for computer vision algorithms, one sample object tracking program was written. It enables Gertie to identify a black rectangle on a white paper. When the paper is moved, Gertie follows the paper with its lamp shade.

The Harris Edge Detection Algorithm [19] is used to identify the four edges of a black rectangle on a white background and their positions within the video frame. Fig. 5 shows the four edges found by the algorithm marked with circles.

The geometrical centre of the rectangle is calculated from the edge positions. Finally a P-controller uses the deviation of the rectangle's center position from the center of the camera's field of vision to command a new position for the lamp shade. The inverse kinematics equations are used to calculate the joint angles for each motor from the cartesian coordinates. That way, the lamp shade always keeps the same orientation and height. It moves in a two dimensional plane, hovering at about $20 \mathrm{~cm}$ above the black rectangle.

Students dedicated to computer vision can use Gertie to test more sophisticated algorithms, which for example recognize and track the hands of someone sitting at a work desk in order to always shed light on the object that person is currently holding in hands.

\section{CONCLUSIONS}

This paper introduced an open hardware platform for conducting research into cognitive products, human-robot interaction or psychology. For example, the paper described two such explorations: the first introducing a simple visual servoing program for tracking illuminated objects; the second exploring the possibility of encouraging intuitive and natural human-robot interaction via replication of emotional body language.

Finally, this paper presented a study on the recognizability of the four emotions displayed by Gertie. While joy and sadness were recognized very reliably with $81 \%$ and $88 \%$ of all people giving the correct answer, fear and surprise were more commonly misinterpreted as surprise and disgust. However, all emotions were recognized above the chance level percentage of $14 \%$. In summary, the study shows that the Robotic Desk Lamp is capable of communicating emotions, despite the fact that it does not dispose of explicit human characteristics.

\section{ACKNOWLEDGMENT}

This research is part of the Innovation@CoTeSys project within the Cluster of Excellence, Cognition for Technical Systems - CoTeSys (www.cotesys.org), funded by the Deutsche Forschungsgemeinschaft (DFG).

\section{REFERENCES}

[1] C. Breazeal, Designing Sociable Robots, ser. Intelligent Robotics and Autonomous Agents. MIT Press, 2004.

[2] S. Sosnowski, A. Bittermann, K. Kuhnlenz, and M. Buss, "Design and evaluation of emotion-display eddie," Proc IEEE/RSJ Int Intelligent Robots and Systems Conf, pp. 3113-3118, 2006.

[3] T. Kuratate, B. Pierce, and G. Cheng, "Mask-bot - a life-size talking head animated robot for av speech and human-robot communication research," in AVSP-2011, 2011, pp. 111-116.

[4] T. Kuratate, Y. Matsusaka, B. Pierce, and G. Cheng, "Mask-bot: a life-size robot head using talking head animation for human-robot communication," in IEEE-RAS International Conference on Humanoid Robots, October 26-28 2011.

[5] A. Beck, L. Canamero, and K. Bard, "Towards an affect space for robots to display emotional body language," in 19th IEEE International Symposium on Robot and Human Interactive Communication, Sept. 12-15 2010, pp. 491-496.

[6] M. Zecca, Y. Mizoguchi, K. Endo, F. Iida, Y. Kawabata, N. Endo, K. Itoh, and A. Takanishi, "Whole body emotion expressions for kobian humanoid robot - preliminary experiments with different emotional patterns," Evaluation, pp. 381-386, 2009.

[7] G. Hoffman, "Ensemble: fluency and embodiment for robots acting with humans," Ph.D. dissertation, Cambridge, MA, USA, 2007.

[8] H. Kozima, M. Michalowski, and C. Nakagawa, "Keepon," International Journal of Social Robotics, vol. 1, pp. 3-18, 2009.

[9] T. Metzler and K. Shea, "Cognitive products: Definition and framework," in 11th International Design Conference, 2010, pp. 865-874.

[10] P. Dario, P. F. Verschure, T. Prescott, G. Cheng, G. Sandini, R. Cingolani, R. Dillmann, D. Floreano, C. Leroux, S. MacNeil, P. Roelfsema, X. Verykios, A. Bicchi, C. Melhuish, and A. Albu-Schäffer, "Robot companions for citizens," Procedia Computer Science, vol. 7, no. 0, pp. 47 - 51, 2011, in Proceedings of the 2nd European Future Technologies Conference and Exhibition 2011 (FET 11).

[11] C. Darwin, The expression of the emotions in man and animals. London: Murray, 1872.

[12] L. R. Caporael and C. M. Heyes, Why anthropomorphize? Folk psychology and other stories. SUNY Press, 1996, ch. 6, pp. 59 -73 .

[13] R. Blake and M. Shiffrar, "Perception of human motion," Annual Review of Psychology, vol. 58, no. July 2006, pp. 47-73, 2007.

[14] J. A. Russell and A. Mehrabian, "Evidence for a three-factor theory of emotions," Journal of Research in Personality, vol. 11, no. 3, pp. 273-294, 1977.

[15] P. Ekman and W. V. Friesen, "The repertoire of nonverbal behavior: Categories, origins, usage, and coding," Semiotica, vol. 1, no. 1, pp. 49-98, 1969.

[16] J. Lasseter, "Principles of traditional animation applied to $3 \mathrm{~d}$ computer animation," SIGGRAPH Comput. Graph., vol. 21, no. 4, pp. 35-44, 1987.

[17] N. Burtnyk and M. Wein, "Interactive skeleton techniques for enhancing motion dynamics in key frame animation," Communications of the $A C M$, vol. 19, no. 10, pp. 564-584, 1976.

[18] A. Billard, S. Calinon, R. Dillmann, and S. Schaal, Handbook of Robotics, 2007, ch. 59: Robot Programming by Demonstration.

[19] G. Bradski and A. Kaehler, Learning OpenCV. O'Reilly Media Inc., 2008. 